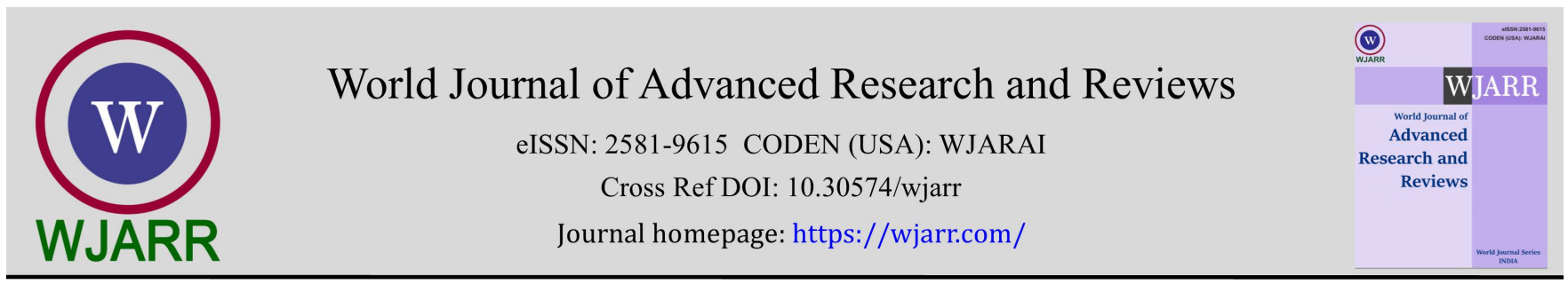

(RESEARCH ARTiCle)

\title{
Assessment of oral bioavailability of nanocapsules loaded-curcumin in-vivo
}

\author{
Ananda ${ }^{1,}{ }^{*}$, Nabeela Tariq ${ }^{1}$, Nosheen Rafiq ${ }^{1}$, Shahzad Yousaf ${ }^{2}$ and Shabbar Abbas ${ }^{2,{ }^{*}}$ \\ ${ }^{1}$ Department of Zoology, Sardar Bahadur Khan Women's University Quetta Balochistan, Pakistan. \\ 2 Department of Biosciciences, COMSATS University Islamabad, Pakistan. \\ 2 Department of Biosciences, COMSATS University Islamabad, Pakistan.
}

World Journal of Advanced Research and Reviews, 2021, 09(02), 005-017

Publication history: Received on 02 January 2021; revised on 09 January 2021; accepted on 11 January 2021

Article DOI: https://doi.org/10.30574/wjarr.2021.9.2.0006

\begin{abstract}
Curcumin, a hydrophobic polyphenol found in rhizome of turmeric (curcuma longa) is one of the generally used spice in Asian countries. It has been found through studies that it has numerous health promoting properties and is proved to have curative properties as it possesses antioxidant and anti-inflammatory properties. Due to low oral bioavailability, decreased solubility, quick metabolism and removal from body, it is challenging to achieve maximum benefits from it. This study was aimed to enhance curcumin oral bioavailability by making use of nanoencapsulation technique. Biodegradable nanocapsules were prepared by consecutive addition of food grade polyelectrolytes including OSAmodified starch (PGU), as an emulsifier, chitosan and sodium-carboxymethylcellulose by ultrasonication technique using primary nanoemulsion as template. Results showed that the mean droplet diameter of nanocapsules was 160.57 $\pm 1.06 \mathrm{~nm}$, the average PDI was $0.14 \pm 0.01$ and average charge was recorded as $-24.43 \pm 0.49 \mathrm{mV}$. Microscopy results showed that the nanostructures were spherical in shape having mean droplet diameter below 200nm. The nanocapsules assessment as a carrier in enhancing the in vivo oral bioavailability of curcumin was made; however, further studies and better tools are needed to clearly know the potential of developed nanosystem.
\end{abstract}

Keywords: Curcumin; Polyelectrolytes; Nanoemulsion; Sonication; Nanocapsules

\section{Introduction}

Bioactive compounds are naturally occurring extra nutritional components present in numerous food sources. They play significant role in improvement of human health and their role in controlling physicochemical reactions in the body is extensively studied. In order to acquire maximum benefits from these compounds, new range of compounds called Nutraceuticals are introduced which besides providing nutrition; provide health benefits such as prevention of degenerative ailments such as cancer [1]. Therefore, the interest in using these products as a preventive measure against numerous diseases has increased dramatically in the recent times hence, the regular consumption of bioactive compounds has gained great importance to prevent and treat diseases for which consumers favor the oral administration compared to other approaches it is less costly, consumer friendly and allow simple dosing plan.

Curcumin is a bioactive compound present in rhizome of turmeric and displays a large number of health benefits. It has anti-inflammatory, antioxidant, anti-aging, antiviral and anti-carcinogenic properties [2-4]. Numerous studies have been carried out in order to unveil its proficiency in treatment and prevention of a number of health issues [5, 6]. Curcumin is proved to be non-toxic to human upon consumption in larger amounts $[7,8]$.

However, like most other bioactive compounds, curcumin has very low bioavailability [9]. The bioavailability of curcumin is negatively affected by decreased solubility $(11 \mathrm{ng} / \mathrm{mL}$ in water), least permeation, rapid metabolism and

\footnotetext{
* Corresponding author: Ananda

Department of Zoology, Sardar Bahadur Khan Women's University Quetta Balochistan, Pakistan.
}

Copyright (c) 2021Author(s) retain the copyright of this article. This article is published under the terms of the Creative Commons Attribution Liscense 4.0. 
removal from the body [10]. After being absorbed by small intestine, curcumin breaks down into its metabolites. Curcumin glucuronide and curcumin sulfate are major metabolites [11].

According to numerous studies, bioavailibity of curcumin has increased upon encapsulation in different materials [12]. Lately, many study groups reported improvement in bioavailability and bioaccessibility of curcumin after encapsulation and delivery via nano-systems [13]. Nanotechnology has gained great importance in the fabrication of efficient delivery system for bioactive compounds in which nanometer sized structures possessing unique properties due to their small size and large surface area are fabricated $[14,15]$. Nanotechnology has positive impact on food science as it improves food texture, taste, processability and increased shelf life [16]. Among wide variety of nanostructures, nanoemulsions are found to be more efficient in the delivery of bioactive compounds [17]. A number of advantages are offered by nanoemulsions including increased bioaccessibility and bioavailability, improved encapsulation efficiencies [15].

On the other hand, these nanoemulsions are thermodynamically unstable hence to overcome the issue and improve its stability, researchers attempted to add ultrathin polymeric layers to the nanodroplets making multilayered droplets [18]. Multilayered nanocapsules are found to have increased permeability for cells resulting in enhanced cellular uptake. They are synthesized from biodegradable materials; they are biologically suitable with body cells and tissues [19]. Efficient encapsulation of bioactive compound is carried out by nanoencapsulation system as the compound is solubilized in the core of polymer hence protecting it from degradation and lowers tissue irritation due to presence of polymeric shell $[20,21]$.

Generally, the evaluation of functional properties of prepared nanostructures is carried out by in-vitro and in-vivo studies. However, the in-vitro models could not mimic complexity of actual GIT hence in-vivo studies were conducted using animal models for the determination of bioavailability of bioactive compound [22, 23]. In-vivo studies provided better understanding of interaction and mechanism of bioactive compound in the body [24]. The present study was conducted with the aim to prepare curcumin-loaded polymeric nanocapsules, its characterization and to assess its oral bioavailability through rat models.

\section{Material and methods}

\subsection{Materials for Nanofabrication}

MCT (NEOBEE 1053) was a caprylic/capric triglyceride (Composition: C8: 55\%, C10: 44\%) and obtained from Stepan Specialty Products Company (Maywood, NJ, USA). Curcumin (catalog number 820354) was supplied by Merck (Darmstadt, Germany) ( $\geq 70.0 \%$ pure, with $\leq 20.0 \%$ of dimethoxy-curcumin and $\leq 5.0 \%$ of bisdemethoxycurcumin). Purity Gum Ultra (PGU), OSA-modified starch, was a gift from Ingredion EMEA (Manchester, UK) and supplied through Rafhan Maize Products, Faisalabad, Pakistan. Low molecular weight chitosan (Aldrich cat. 448869, degree of deacetylation: > 85\%, made from shrimp shells) was purchased from Sigma-Aldrich Company Ltd. Carboxymethyl cellulose (Na-CMC, Mw- 90,000 kDa, Sigma-Aldrich: 419273), a biodegradable polymer with 50-200 cps viscosity for $4 \% \mathrm{w} / \mathrm{v}$ aqueous solution. Other chemicals/reagents used were of analytical grade and obtained from Merck (Darmstadt, Germany). Doubly distilled water was used for all solutions and in nanoemulsion preparation.

\subsection{Materials In vivo Studies}

Twelve rats (Sprague Dawley) of 8-12 weeks age were purchased and acclimatized at animal house, National Institute of Health (NIH), Islamabad.

\subsection{Methods}

Emulsifier (PGU) solution was prepared by dispersing $1.5 \% \mathrm{w} / \mathrm{v}$ of amorphous PGU in warm water at $45^{\circ} \mathrm{C}$ followed stirring for 30 minutes. Acetate buffer $(0.1 \mathrm{M}, 4.5 \mathrm{pH})$ solution was prepared by dissolving $3.15 \mathrm{~g}$ of sodium acetate in water followed by the addition of glacial acetic acid. Acetate buffer solution was used to prepare $0.075 \%(\mathrm{w} / \mathrm{v})$ chitosan solution by overnight stirring.

CMC dispersion $(0.1 \% \mathrm{w} / \mathrm{v})$ was prepared by dissolving CMC in distilled water and stirred for 1 minute.

\subsection{Preparation of nanostructures}

Oil phase was prepared by dispersing curcumin $(6 \mathrm{mg} / \mathrm{mL})$ in MCT oil for which oil was preheated at $100^{\circ} \mathrm{C}$ for $5 \mathrm{~min}$ and curcumin was added in to it and stirred for further 2 minutes by hot plate stirrer as demonstrated by [25]. Curcumin 
enriched MCT oil was cooled down at room temperature and stored in dark place for further experiments. For the preparation of aqueous phase, $1.5 \% \mathrm{w} / \mathrm{v}$ of emulsifier was dissolved at $50^{\circ} \mathrm{C}$ for 30 minutes using magnetic stirrer.

Firstly, $5 \mathrm{~mL}$ oil phase and $95 \mathrm{~mL}$ aqueous phase were homogenized coarsely using a high-speed blender (Yellow Line DI basic homogenizer) at $13500 \mathrm{rpm}$ for 2 minutes to prepare coarse emulsion which was followed by sonication (Ultra sonic processor model CV18 of Cole Parmer Company) at $20 \mathrm{kHz}$ operating frequency for 10 minutes. The temperature of sample was maintained at $45^{\circ} \mathrm{C}$ by cold water.

\subsection{Preparation of multilayer nanoemulsion}

Primary nanoemulsions NEI was used as a template for the preparation of nanocapsules by sequential deposition of chitosan and CMC polyelectrolytes. NEII were prepared by the addition of Chitosan dispersion $(10 \mathrm{~mL})$ drop wise into the $20 \mathrm{~mL}$ of NEI, and coarsely homogenized for $5 \mathrm{~min}$. It was further ultrasonically homogenized for 7 minutes to acquire the double layered nanoemulsions or NEII. Nanocapsules were prepared by adding $6.66 \mathrm{~mL}$ of CMC solution dropwise in $20 \mathrm{~mL}$ of NEII and homogenized for $5 \mathrm{~min}$ using high speed blender followed by sonication for $3 \mathrm{~min}$ to obtain triple layered nanocapsules suspension. Flow diagram of the whole preparation process of nanocapsules suspension is given in Figure 1.

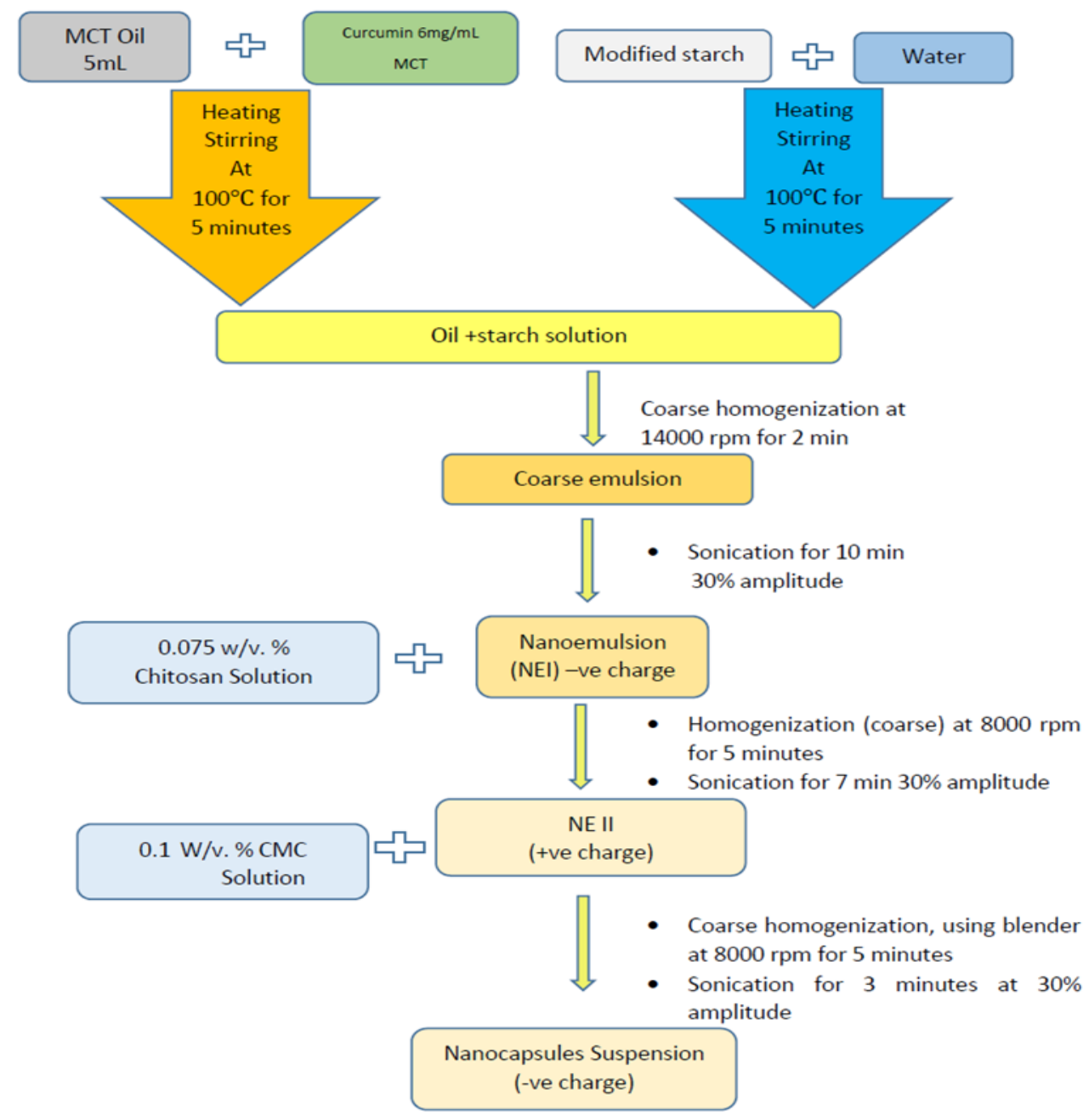

Figure 1 Flow diagram of the preparation of nanocapsules

\subsection{Measurement of droplet size and zeta potential}

Mean droplet diameter (MDD) and polydispersity index and charge of nanostructures investigated in triplicate by using Zetasizer (Malvern Instruments, Ltd., UK) equipped with dynamic light scattering technology [18]. Samples were diluted 200 -folds using purified water. Surface charge of the nanostructures was checked by measuring electrophoretic mobility at $25^{\circ} \mathrm{C}$. The size (MDD) was expressed in $\mathrm{nm}$ and charge in $\mathrm{mV}$ [25]. 


\subsection{Microscopy}

Microscopy was conducted for nanostructures using light microscope (NIKON, model YS 100). Samples were observed at magnifying power of $10 \mathrm{X}$ and $40 \mathrm{X}$ at room temperature.

\subsection{Transmission electron microscopy (TEM)}

Electron microscope, JOEL JEM-7000 transmission electron microscope was employed at accelerating voltage of $80 \mathrm{kV}$ for morphological study of prepared nanocapsules. Briefly, nanocapsule suspension were put on the carbon coated copper grid and stained with $2 \%$ phosphotungstic acid and dried at room temperature before analysis [26].

\subsection{In vivo Studies}

Twelve Sprague Dawley (250-300g, 8-12weeks) rats were used to determine the oral bioavailability of curcumin [27]. Rats were divided in 4 groups under $12 \mathrm{~h}$ light/dark cycle at $22-26^{\circ} \mathrm{C}$ and $50 \%$ humidity. After a week of adaptation, rats were fasted overnight and a total of $16 \mathrm{~mL}$ of control (CMC solution) NE I, NE II, and nanocapsules (4mL each) given at intervals of 30 minutes, were administered in their stomach. Rats were anesthetized with chloroform after $30 \mathrm{~min}$. and sacrificed for blood collection. Blood samples for plasma were collected in heparinized vials.

\subsection{Plasma separation}

Blood samples were centrifuged at $4000 \mathrm{~g}$ for 10 minutes at $4{ }^{\circ} \mathrm{C}$ to separate the plasma. Plasma samples were stored at $-20^{\circ} \mathrm{C}$ for further analysis.

Extraction: Curcumin was extracted from plasma samples by adding $0.1 \mathrm{~mL}$ acetonitrile into $0.1 \mathrm{~mL}$ plasma and centrifuged at $4000 \mathrm{rpm}$ for $5 \mathrm{~min}$. The supernatant was collected and subjected to further analysis by HPLC [28].

\subsection{HPLC}

HPLC analysis was performed using Shimadzu LC-20A (Kyoto, Japan) HPLC system, consisting the UV-Visible indicator supplied with C8 column $(4.6 \times 150 \mathrm{~mm}, 3 \mu \mathrm{m})$ with guard column $(4.6 \times 20 \mathrm{~mm}, 3 \mu \mathrm{m})$, kept at $35^{\circ} \mathrm{C}$. The mobile phase was composed of two components: (A) $10 \mathrm{mM}$ of ammonium acetate buffer solution with adjusted $\mathrm{pH} 4.5$ and (B) acetonitrile. The rate of flow was set to be $1.5 \mathrm{~mL} /$ minute whereas, the UV-detector was adjusted at $426 \mathrm{~nm}$. Its elution was gradient elution starting with $95 \% \mathrm{~A}$ and $5 \% \mathrm{~B}$, to be changed t0 55\% A and $45 \% \mathrm{~B}$ at 20 min then changed to $5 \%$ $\mathrm{A}$ and $95 \% \mathrm{~B}$ at $33 \mathrm{~min}[28]$

\subsection{Statistical Analysis}

All statistical analysis was done using MS-EXCEL-2013 package. All measurements were done for at least three replicates. Results are presented as means \pm standard deviation of means.

\section{Results}

The curcumin nanoemulsion and nanocapsules prepared by coarse homogenization followed by ultra-sonication treatment were characterized for their size (mean droplet diameter or MDD), polydispersity index (PDI) and surface charge using Zetasizer ZS nano (Malvern Instruments, Ltd., UK). Furthermore, their morphological studies were conducted by conventional light microscopy and TEM. Finally, blood plasma was attempted to determine through high performance liquid chromatography (HPLC) for curcumin concentration.

\subsection{The MMD, PDI and Zeta Potential}

Mean droplet diameter (MDD) is the average of hydrodynamic size of a droplet or particle while polydispersity index (PDI) is the measure of distribution of molecular mass in a sample of polymer. It measures the deviation from the uniformity of a dispersion. Zeta Potential is the charge present on the surface of the droplets.

The MDD, PDI and Zeta Potential NE I, NE II and Nanocapsules are summarized in Table 1. 
Table 1 MDD, PDI and Zeta potential of primary, secondary and multilayered nanoemulsions

\begin{tabular}{|l|l|l|l|}
\hline Sample & MDD & PDI & Zeta-Potential \\
\hline NE I & $142.97 \pm 2.51$ & $0.18 \pm 0.01$ & $-36.13 \pm 1.70$ \\
\hline NE II & $151.03 \pm 5.76$ & $0.16 \pm 0.02$ & $+09.30 \pm 0.49$ \\
\hline NE III & $160.57 \pm 1.06$ & $0.14 \pm 0.01$ & $-24.43 \pm 0.49$ \\
\hline
\end{tabular}

\subsection{Curcumin Loaded Single-Layer Nanoemulsion (NE I)}

The sample containing curcumin-loaded nanostructures was analyzed in triplicate and the result was recorded in terms of MDD. Each sample was represented by an individual peak, and their MDD was found to be $144.8 \pm 2.51 \mathrm{~nm}$ (Figure2). The average PDI of these samples was recorded as $0.18 \pm 0.01$. The surface charge on the starch stabilized nanodroplets was negative. Results were recorded in triplicate and the average negative charge present on the droplets was determined as $-36.13 \pm 1.70 \mathrm{mV}$, as shown in Figure-3.

$\begin{array}{rllll} & & \text { Size (d.nm): } & \text { \% Intensity: } & \text { St Dev (d.nm): } \\ \text { Z-Average (d.nm): } 144.8 & \text { Peak 1: } & 173.1 & 100.0 & 80.17 \\ \text { Pdl: } 0.172 & \text { Peak 2: } & 0.000 & 0.0 & 0.000 \\ \text { Intercept: } 0.883 & \text { Peak 3: } & 0.000 & 0.0 & 0.000 \\ \text { Result quality: Good } & & & & \end{array}$

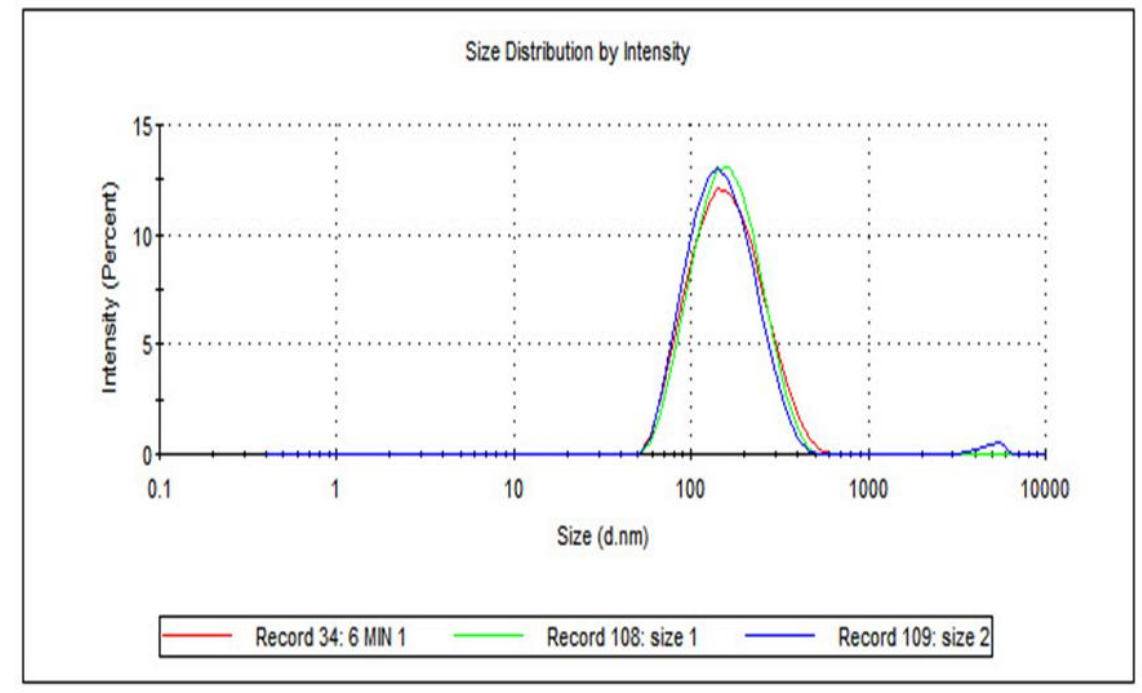

Figure 2 The MDD and PDI of curcumin-loaded nanodroplets (NE I)

\subsection{Curcumin Loaded Double-Layer Nanoemulsion (NE II)}

The sample was examined three times and result was presented in MDD. The MDD of the sample droplet was found to be $151.03 \pm 5.76 \mathrm{~nm}$ and the average PDI was recorded as $0.16 \pm 0.02$ (Figure-4). There was a net positive charge on the surface of double layer nano-droplet. The average positive charge present on the droplets was $9.3 \pm 0.49 \mathrm{mV}$ (Figure5). 
World Journal of Advanced Research and Reviews, 2021, 09(02), 005-017

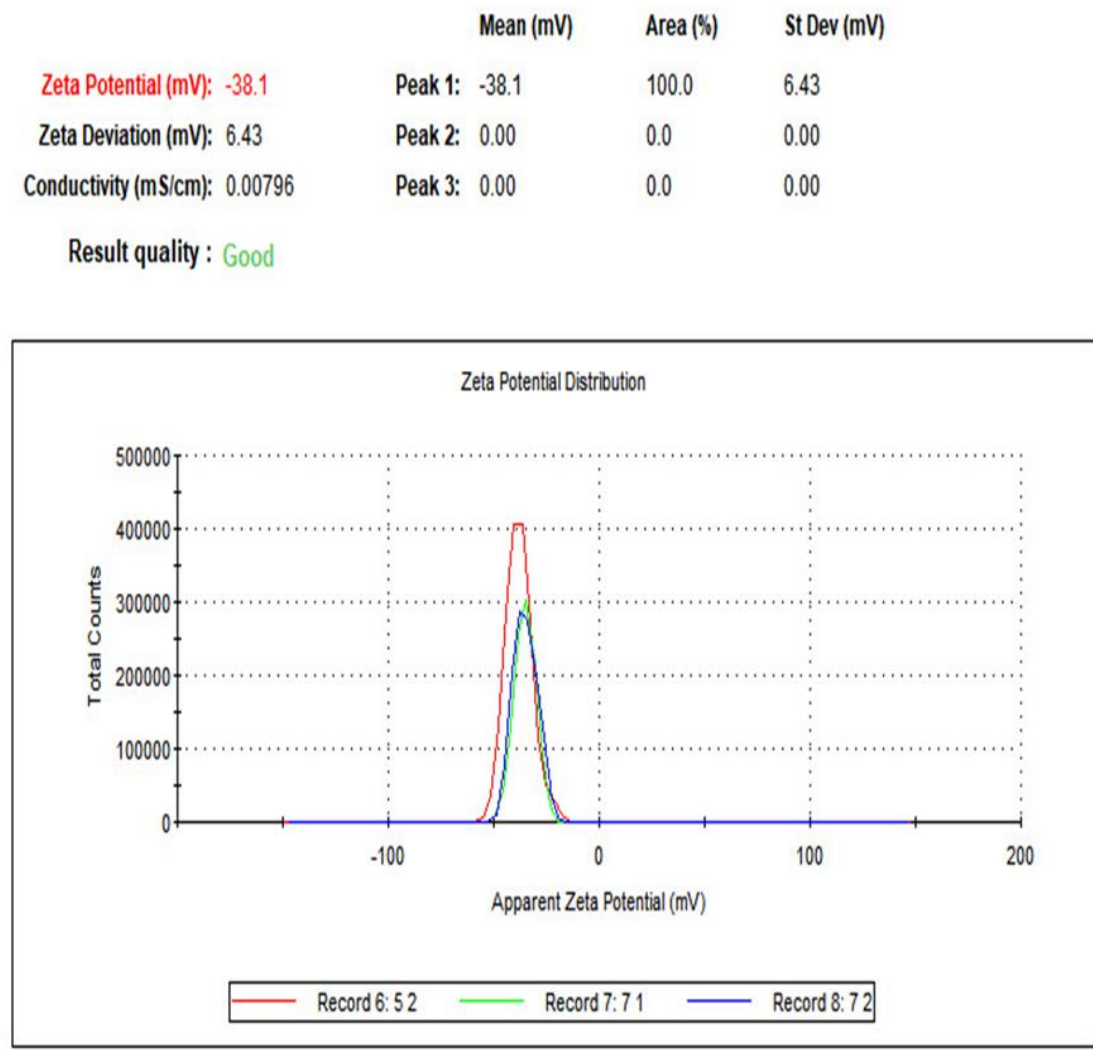

Figure 3 Charge present on the primary nano-droplets NE I

$\begin{array}{rllll} & & \text { Size (d.nm): } & \text { \% Intensity: } & \text { St Dev (d.nm): } \\ \text { Z-Average (d.nm): } 154.5 & \text { Peak 1: } & 181.7 & 100.0 & 76.22 \\ \text { Pdl: } 0.139 & \text { Peak 2: } & 0.000 & 0.0 & 0.000 \\ \text { Intercept: } 0.883 & \text { Peak 3: } & 0.000 & 0.0 & 0.000 \\ \text { Result quality: Good } & & & & \end{array}$

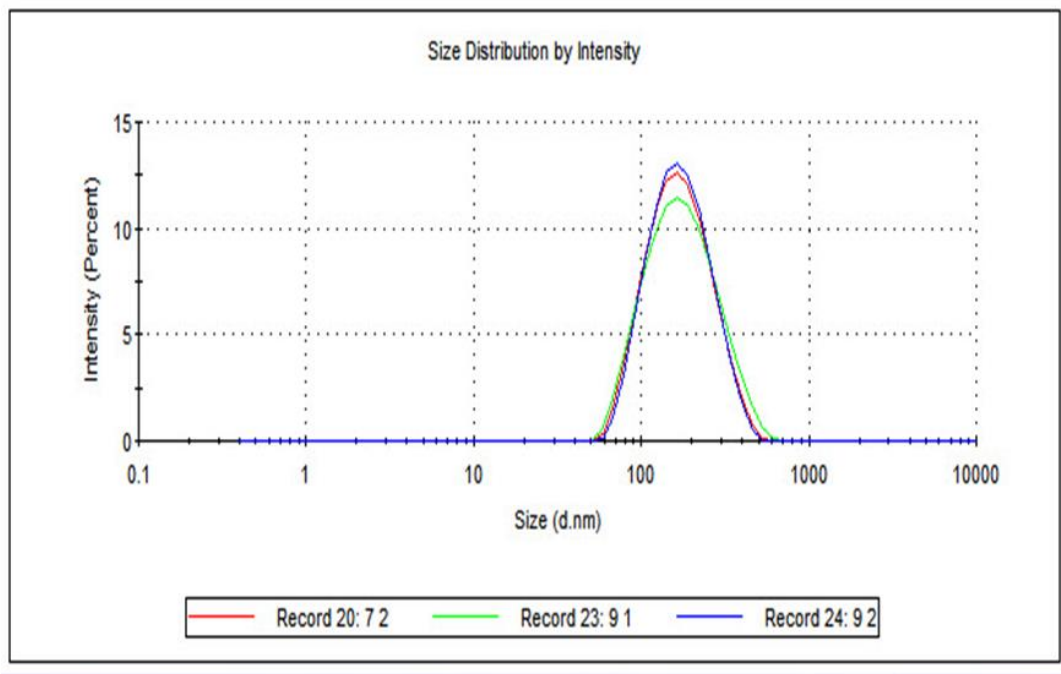

Figure 4 The MDD and PDI of secondary nano-droplets 


\begin{tabular}{|c|c|c|c|c|}
\hline & & Mean $(\mathrm{mV})$ & Area $(\%)$ & St Dev (mV) \\
\hline Zeta Potential (mV): 8.74 & Peak 1: & 8.74 & 100.0 & 3.80 \\
\hline Zeta Deviation $(\mathrm{mV}): 3.80$ & Peak 2: & 0.00 & 0.0 & 0.00 \\
\hline Conductivity (mS/cm): 0.0362 & Peak 3: & 0.00 & 0.0 & 0.00 \\
\hline
\end{tabular}

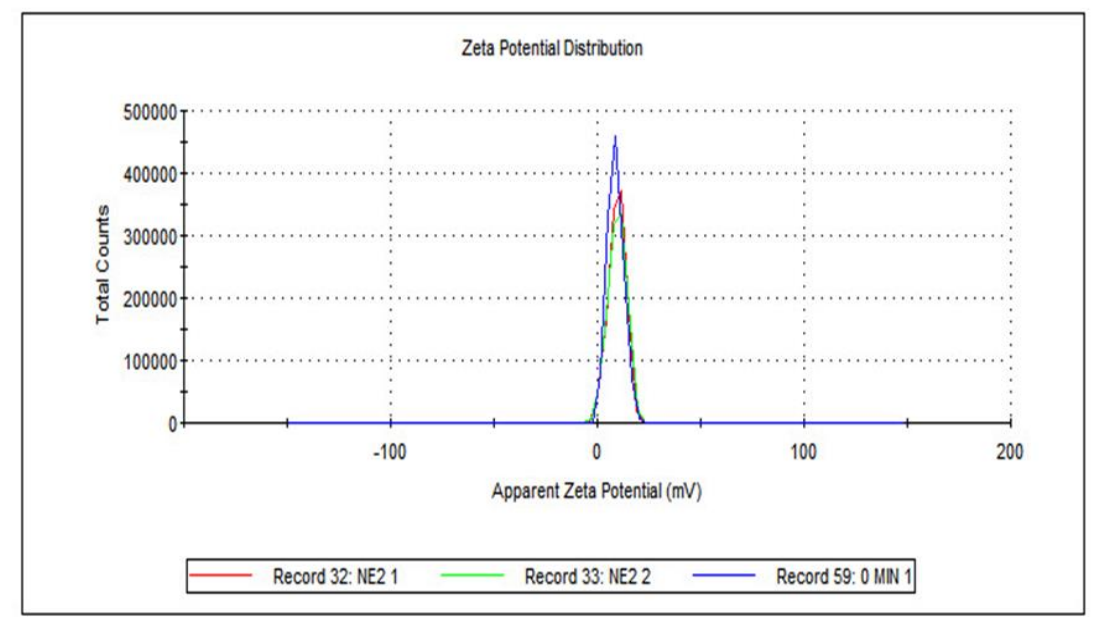

Figure 5 Charge present on the surface of secondary nano-droplets

\subsection{Curcumin Loaded Nanocapsules (NE III)}

The MDD of nanocapsules was recorded as $160.57 \pm 1.06 \mathrm{~nm}$. The mean PDI value of these nanocapsules was $0.14 \pm$ 0.01 (Figure-6). The net surface charge on the nanocapsules was negative. The average zeta potential was $-24.43 \pm 0.49$ $\mathrm{mV}$, as presented in Figure-7.

$\begin{array}{rllll} & & \text { Size (d.nm): } & \text { \% Intensity: } & \text { St Dev (d.nm): } \\ \text { Z.Average (d.nm): } 160.4 & \text { Peak 1: } & 189.0 & 100.0 & 76.57 \\ \text { Pdll: } 0.144 & \text { Peak 2: } & 0.000 & 0.0 & 0.000 \\ \text { Intercept: } 0.905 & \text { Peak 3: } & 0.000 & 0.0 & 0.000 \\ \text { Result quality: Good } & & & & \end{array}$

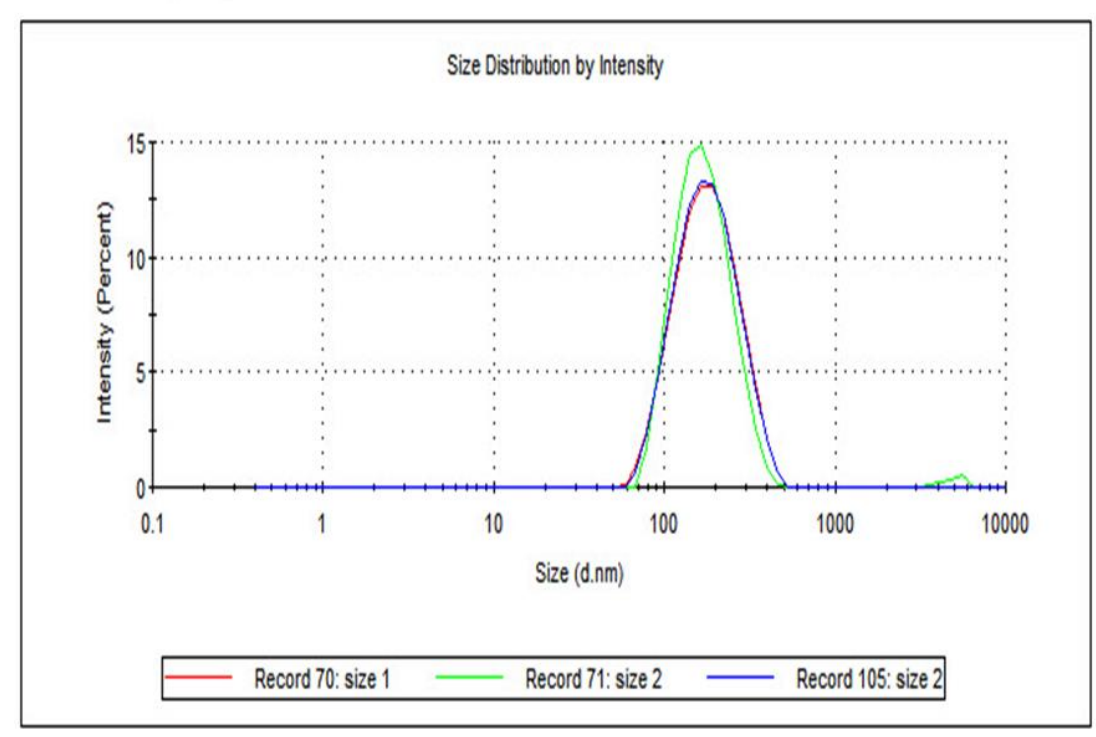

Figure 6 MDD and PDI of nanocapsules 


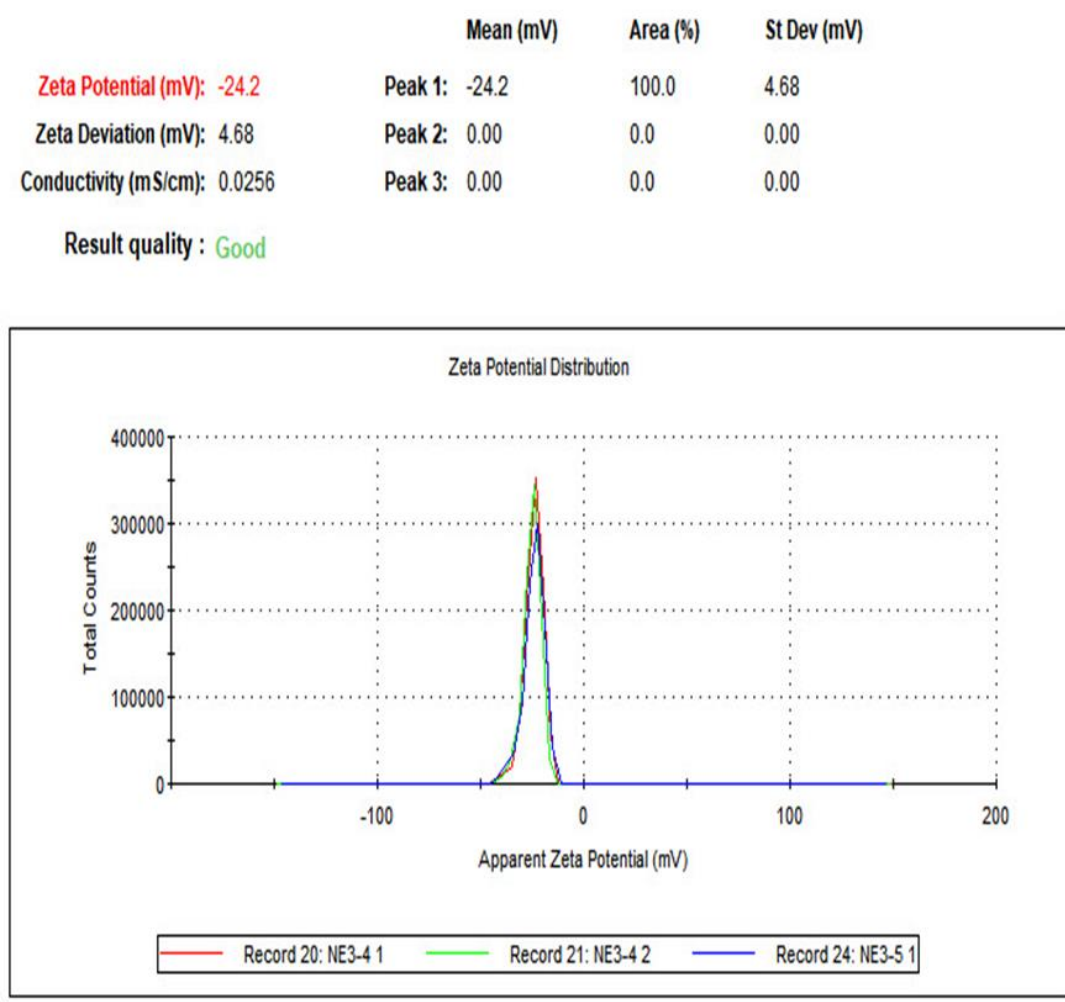

Figure 7 Charge present on the nanocapsule surface

\subsection{Microscopy}

Microscopy of the samples was done in order to visualize the nanodroplets and nanocapsules and study their morphology.

\subsection{Light Microscopy}

Light microscopy of the coarse emulsion, nanoemulsions and nanocapsules was conducted using compound microscope. Only coarse emulsions were visible under simple microscope due to their micron (Figure-8a). Nanoemulsions and nanocapsules could not be seen through light microscopy as nanoscale material is difficult to observe (see Figure 8b, c and d).

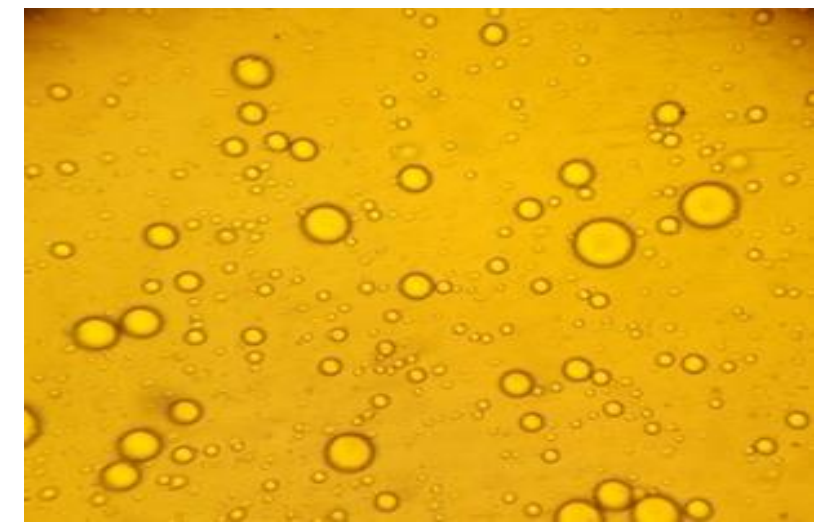

A

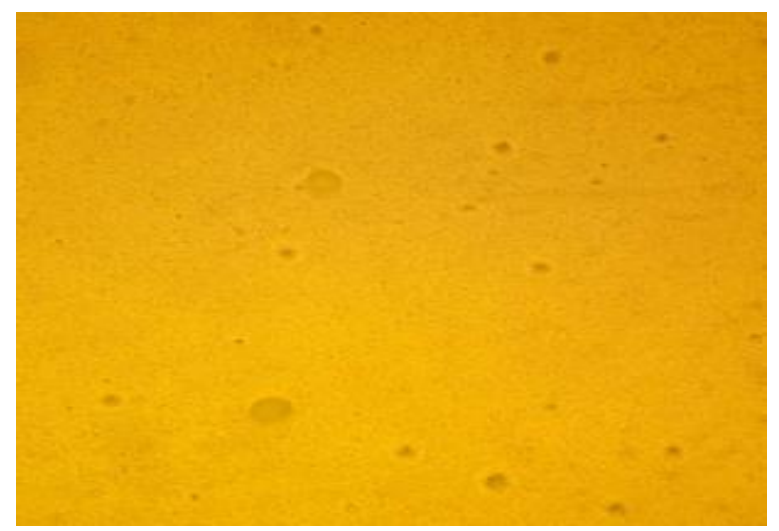

B 


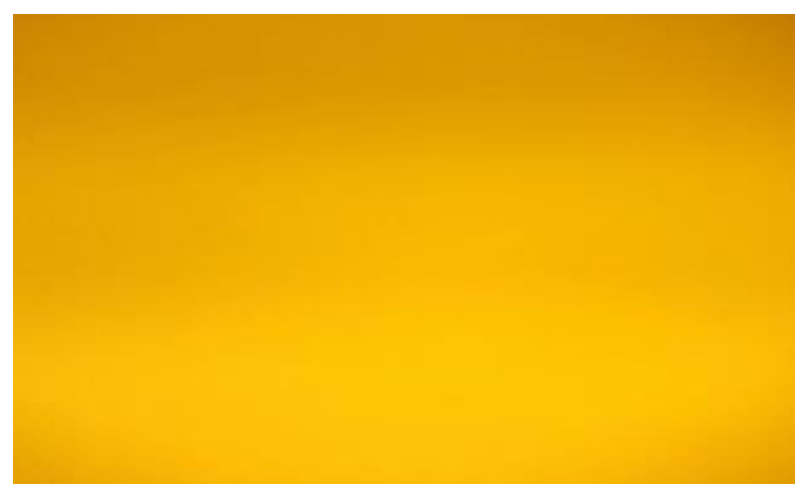

C

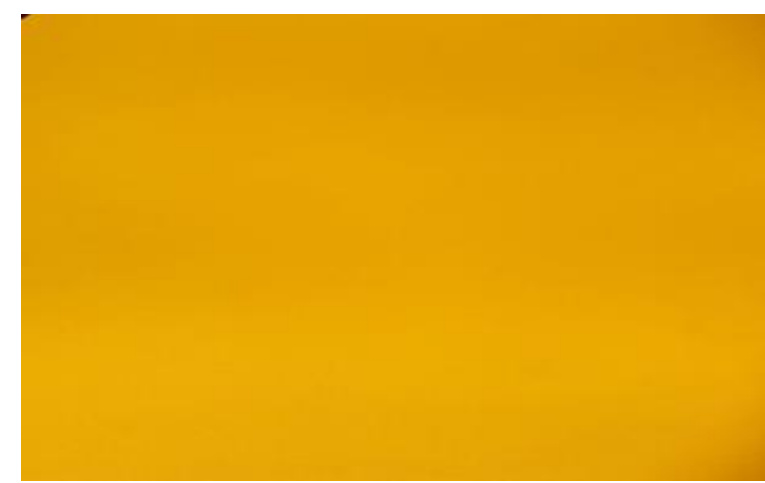

D

Figure 8 A- Light microscopy of coarse emulsion; B- Light microscopy of NE I; C- Light microscopy of NE II D- Light microscopy of NE III

\subsection{Transmission Electron Microscopy (TEM)}

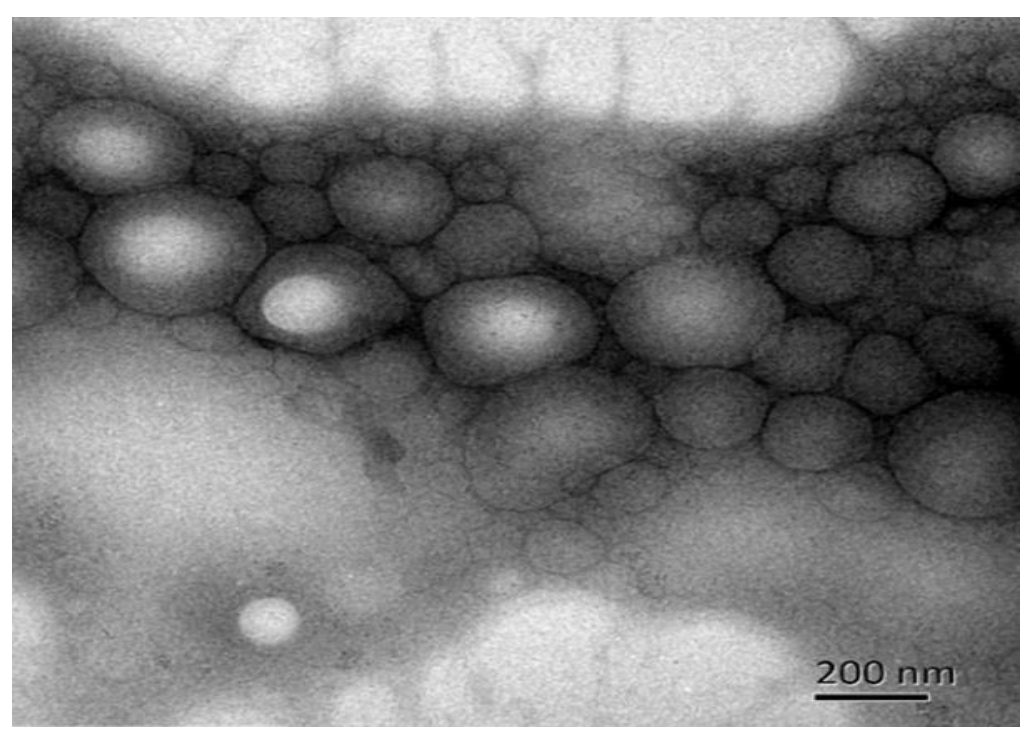

Figure 9 TEM image of nanocapsules showing the morphology and the core material

The morphology of nanocapsules was determined through TEM analysis and according to it, the average number of the nanocapsules had lower than $200 \mathrm{~nm}$ sizes and these core-shell structures are spherical in shape. The core material was also visible through the thick outer layer as core part appeared to be lighter and the outer layers appeared darker in color.

\subsection{In-vivo Absorption Study}

Quantification of curcumin present in blood plasma was conducted by HPLC method in order to assess the absorption of curcumin loaded in nanocapsules. In the first step, standard chromatogram was obtained for pure curcumin compound, as shown in Figure 12a. 


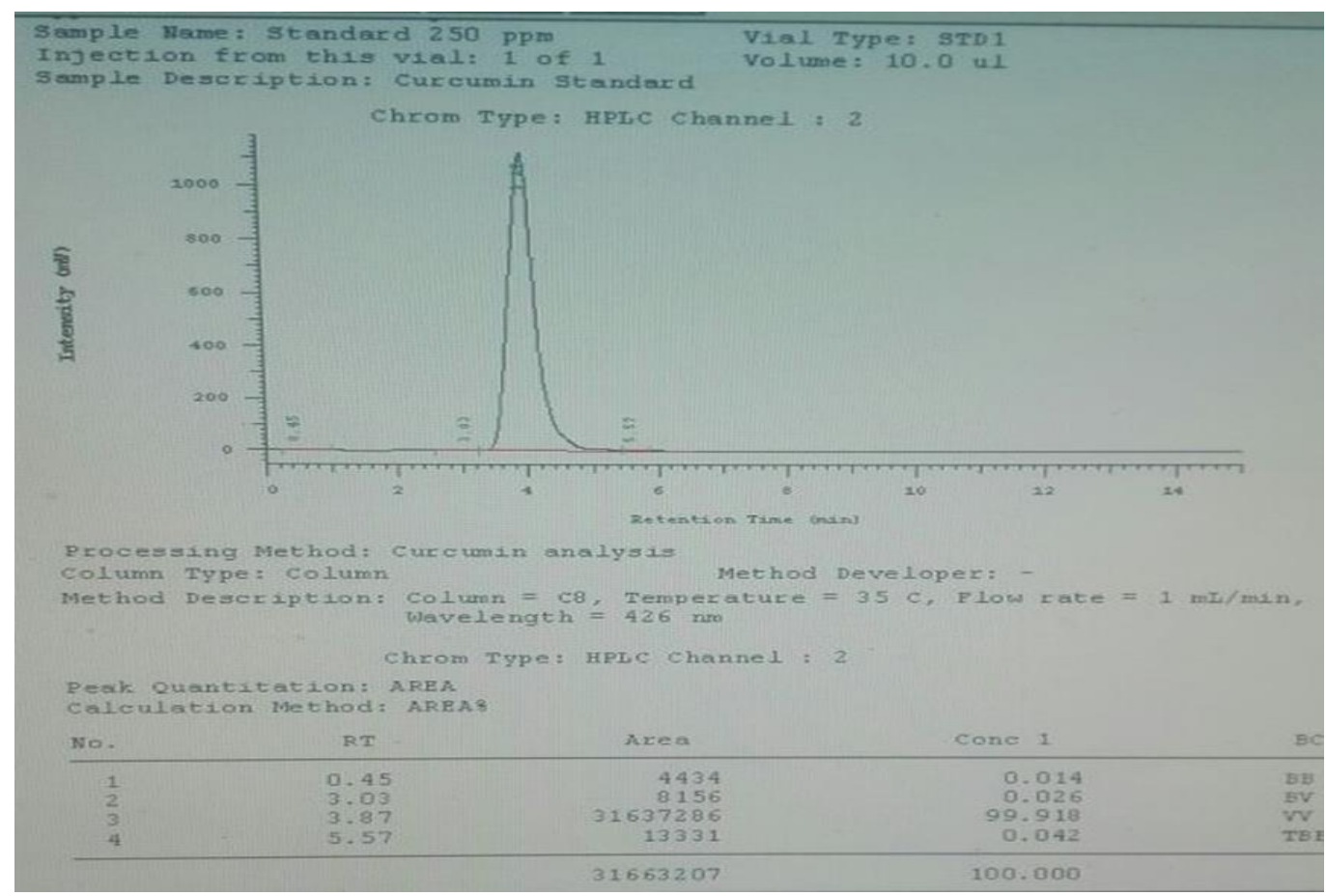

Figure 10a A chromatogram showing standard curcumin HPLC profile

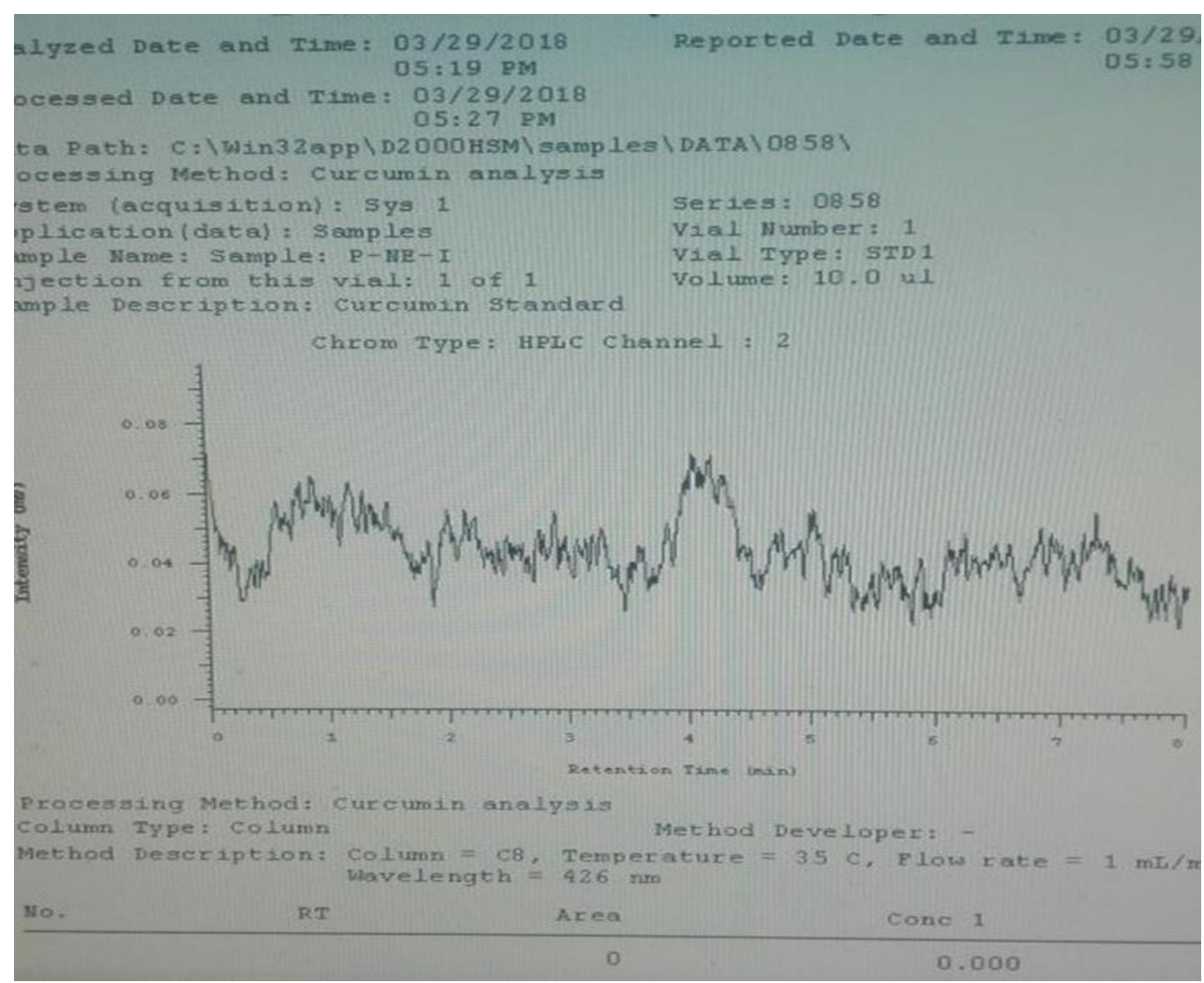

Figure 10b Chromatogram of plasma sample obtained from a rat which was orally administered with curcumin loaded nanocapsule suspension

As no clear chromatographic peaks (in the area of interest) were obtained in the case of blood plasma obtained from the rats administered (orally) with NEI and NEII, their results are excluded from the current study. 


\section{Discussion}

Curcumin, an active component of turmeric that has been used as a food spice and homeopathic medication since long. It found to have preventive and curative properties in numerous diseases due to its antioxidant and anti-inflammatory properties [29]. Due to several challenges like low bioavailability, solubility, rapid metabolism and quick removal from the body its potential benefits cannot be achieved.

In this study, nanotechnology was used to overcome the issue related to curcumin. Firstly, o/w nanoemulsions were prepared by ultrasonication and were further used as template for nanocapsule fabrication. Numerous studies suggest that nanostructures with size range of 20-200nm have increased stability against physical destabilization [30-32]. In our study, the prepared nanostructures had size below $200 \mathrm{~nm}$.

The mean diameter of nanocapsules was recorded $160.57 \pm 1.06 \mathrm{~nm}$ which was in accordance with [18] in which the mean diameter of nanocapsule was $159.85 \pm 0.92 \mathrm{~nm}$. Likewise, Mishra et al. [33] prepared stable multilayered nanoemulsion using layer-by-layer technology, and particle size were in range of 90.8 to $167.8 \mathrm{~nm}$. Many studies found that stability of encapsulated compound against oxidation was improved in multilayer nanoemulsions [34].

The PDI value below 0.2 is considered reliable as it indicates the uniformity in size of nanostructures. The nanostructures prepared in our study had average PDI value ranging from $0.14 \pm 0.01$ to $0.18 \pm 0.01$.

The surface charge (expressed in $\mathrm{mV}$ ) on the nanostructures is an essential parameter in maintaining stability of the system by their electrostatic behavior. In case, particles are highly positive/negative, strong repulsion will occur and if they are weakly charged, coalescence occurs, hence declining system's stability. Zanotto-Filho et al. [22] prepared multilayer nanocapsules having the zeta potential value of -9.56 with enhanced efficiency. The mean charge value on the particles prepared in our study was recorded as $-24.43 \pm 0.49 \mathrm{mV}$ which is somewhat higher that recorded by Abbas et al., 2015, i.e., $-17.2 \pm 0.35 \mathrm{mV}$ [22]. Similar results were recorded by Mishra et al. [33] and Langella et al. [35] with the zeta potential values of 22.9 to $31.01 \mathrm{mV}$.

TEM gives information on the morphology of the prepared nanostructures i.e. size, shape, and wall thickness [36]. According to the TEM results, the particles had spherical shape and outer shell could be differentiated form inner core. Spherical shape provides surface area and mobility hence assisting in absorption of bioactive compound being loaded.

HPLC was conducted to assess the oral bioavailability of encapsulated bioactive.

However, a compound peak was obtained (Figure 10b), which indicates that there was interference of other compounds (usually proteins) as well. As a result, the quantification of curcumin was almost impossible. However, the presence of curcumin in the plasma samples of rat is indicated by the chromatograph. Nanocapsules helped in raising the level of curcumin in the blood. Curcumin was not detected from plasma of rats which were fed on NEI and NEII by this method.

The discussion is concluded with statement that "curcumin could not be detected using conventional methods as probably it was reduced and converted into metabolites and its actual concentration became too low for detection.

\section{Conclusion}

In this study, nanostructures including curcumin-loaded polymeric nanocapsules using high energy method (ultrasonication) were successfully prepared. As all three type of nanostructures were prepared from food grade material, they could be good candidates as a vehicle for the oral delivery of nutrients including curcumin. Due to poor water-solubility and chemical stability, curcumin bioavailability is significantly compromised. In our study, only nanocapsules (triple-layered structures) showed increased oral bioavailability of curcumin. However further study is recommended to exactly quantify the curcumin concentration in blood plasma.

\section{Compliance with ethical standards}

\section{Acknowledgments}

I thankfully acknowledge HEC Pakistan for financial support under the program "Strengthening and Development of Sardar Bahadur Khan Women's University, Quetta” for the year 2017 to 2018. 


\section{Disclosure of conflict of interest}

The authors declare that they have no conflict of interest.

\section{Statement of ethical approval}

Animals used in this study were handled, according to ethical guidelines of COMSATS University Islamabad, Pakistan.

\section{References}

[1] Go VL, Harris DM, Srihari P. Global overview of the role of nutraceuticals in cancer. Nutraceuticals and Cancer. 2012; 1-10.

[2] Garcea G, Berry DP, Jones DJ, Singh R, Dennison AR, Farmer PB, Sharma RA, Steward WP, Gescher AJ. Consumption of the putative chemopreventive agent curcumin by cancer patients: assessment of curcumin levels in the colorectum and their pharmacodynamic consequences. Cancer Epidemiology and Prevention Biomarkers. 2005; 14(1): 120-25.

[3] Sharma RA, Gescher AJ, Steward WP. Curcumin: the story so far. European journal of cancer. 2005; 41(13): 195568.

[4] Hanai H, Iida T, Takeuchi K, Watanabe F, Maruyama Y, Andoh A, Tsujikawa T, Fujiyama Y, Mitsuyama K, Sata M, Yamada M. Curcumin maintenance therapy for ulcerative colitis: randomized, multicenter, double-blind, placebocontrolled trial. Clinical Gastroenterology and Hepatology. 2006; 4(12): 1502-6.

[5] Brietzke E, Mansur RB, Zugman A, Carvalho AF, Macêdo DS, Cha DS, Abílio VC, McIntyre RS. Is there a role for curcumin in the treatment of bipolar disorder?. Medical Hypotheses. 2013; 80(5): 606-12.

[6] Çelik A, Eke D, Ekinci SY, Yıldırım S. The protective role of curcumin on perfluorooctane sulfonate-induced genotoxicity: single cell gel electrophoresis and micronucleus test. Food and chemical toxicology. 2013; 53: 24955 .

[7] Hsieh CY. Phase I clinical trial of curcumin, a chemopreventive agent, in patients with high-risk or pre-malignant lesions. Anticancer Res. 2001; 21(2895): e2900.

[8] Sharma RA, McLelland HR, Hill KA, Ireson CR, Euden SA, Manson MM, Pirmohamed M, Marnett LJ, Gescher AJ, Steward WP. Pharmacodynamic and pharmacokinetic study of oral Curcuma extract in patients with colorectal cancer. Clinical Cancer Research. 2001; 7(7): 1894-900.

[9] Anand P, Kunnumakkara AB, Newman RA, Aggarwal BB. Bioavailability of curcumin: problems and promises. Molecular pharmaceutics. 2007; 4(6): 807-18.

[10] Kaminaga Y, Nagatsu A, Akiyama T, Sugimoto N, Yamazaki T, Maitani T, Mizukami H. Production of unnatural glucosides of curcumin with drastically enhanced water solubility by cell suspension cultures of Catharanthus roseus. FEBS letters. 2003; 555(2): 311-6.

[11] Aggarwal BB, Sung B. Pharmacological basis for the role of curcumin in chronic diseases: an age-old spice with modern targets. Trends in pharmacological sciences. 2009; 30(2): 85-94.

[12] Kakkar V, Singh S, Singla D, Kaur IP. Exploring solid lipid nanoparticles to enhance the oral bioavailability of curcumin. Molecular nutrition \& food research. 2011; 55(3): 495-503.

[13] Ahmed K, Li Y, McClements DJ, Xiao H. Nanoemulsion-and emulsion-based delivery systems for curcumin: Encapsulation and release properties. Food Chemistry. 2012; 132(2): 799-807.

[14] Acosta E. Bioavailability of nanoparticles in nutrient and nutraceutical delivery. Current opinion in colloid \& interface science. 2009; 14(1): 3-15.

[15] Huang Q, Yu H, Ru Q. Bioavailability and delivery of nutraceuticals using nanotechnology. Journal of food science. 2010; 75(1): R50-7.

[16] Duncan TV. Applications of nanotechnology in food packaging and food safety: barrier materials, antimicrobials and sensors. Journal of colloid and interface science. 2011; 363(1): 1-24.

[17] McClements DJ. Food emulsions: principles, practices, and techniques. CRC press. 2015. 
[18] Abbas S, Karangwa E, Bashari M, Hayat K, Hong X, Sharif HR, Zhang X. Fabrication of polymeric nanocapsules from curcumin-loaded nanoemulsion templates by self-assembly. Ultrasonics sonochemistry. 2015; 23 : 81-92.

[19] Mosqueira VC, Legrand P, Morgat JL, Vert M, Mysiakine E, Gref R, Devissaguet JP, Barratt G. Biodistribution of long-circulating PEG-grafted nanocapsules in mice: effects of PEG chain length and density. Pharmaceutical research. 2001; 18(10): 1411-9.

[20] Reis CP, Neufeld RJ, Ribeiro AJ, Veiga F. Nanoencapsulation I. Methods for preparation of drug-loaded polymeric nanoparticles. Nanomedicine: Nanotechnology, Biology and Medicine. 2006; 2(1): 8-21.

[21] Anton N, Benoit JP, Saulnier P. Design and production of nanoparticles formulated from nano-emulsion templates-a review. Journal of controlled release. 2008; 128(3): 185-99.

[22] Zanotto-Filho A, Coradini K, Braganhol E, Schröder R, De Oliveira CM, Simões-Pires A, Battastini AM, Pohlmann AR, Guterres SS, Forcelini CM, Beck RC. Curcumin-loaded lipid-core nanocapsules as a strategy to improve pharmacological efficacy of curcumin in glioma treatment. European Journal of Pharmaceutics and Biopharmaceutics. 2013; 83(2): 156-67.

[23] Peng S, Li Z, Zou L, Liu W, Liu C, McClements DJ. Enhancement of curcumin bioavailability by encapsulation in sophorolipid-coated nanoparticles: an in vitro and in vivo study. Journal of agricultural and food chemistry. 2018; 66(6): 1488-97.

[24] Peng S, Li Z, Zou L, Liu W, Liu C, McClements DJ. Improving curcumin solubility and bioavailability by encapsulation in saponin-coated curcumin nanoparticles prepared using a simple pH-driven loading method. Food \& function. 2018; 9(3): 1829-39.

[25] Abbas S, Bashari M, Akhtar W, Li WW, Zhang X. Process optimization of ultrasound-assisted curcumin nanoemulsions stabilized by OSA-modified starch. Ultrasonics sonochemistry. 2014; 21(4): 1265-74.

[26] Tan C, Feng B, Zhang X, Xia W, Xia S. Biopolymer-coated liposomes by electrostatic adsorption of chitosan (chitosomes) as novel delivery systems for carotenoids. Food hydrocolloids. 2016; 52: 774-84.

[27] Maiti K, Mukherjee K, Gantait A, Saha BP, Mukherjee PK. Curcumin-phospholipid complex: preparation, therapeutic evaluation and pharmacokinetic study in rats. International journal of pharmaceutics. 2007; 330(12): 155-63.

[28] Allam AN, Komeil IA, Fouda MA, Abdallah OY. Preparation, characterization and in vivo evaluation of curcumin self-nano phospholipid dispersion as an approach to enhance oral bioavailability. International Journal of Pharmaceutics. 2015; 489(1-2): 117-23.

[29] Ghalandarlaki N, Alizadeh AM, Ashkani-Esfahani S. Nanotechnology-applied curcumin for different diseases therapy. BioMed research international. 2014.

[30] Solans C, Izquierdo P, Nolla J, Azemar N, Garcia-Celma MJ. Nano-emulsions. Current opinion in colloid \& interface science. 2005; 10(3-4): 102-10.

[31] Wooster TJ, Golding M, Sanguansri P. Impact of oil type on nanoemulsion formation and Ostwald ripening stability. Langmuir. 2008; 24(22): 12758-65.

[32] Sagalowicz L, Leser ME. Delivery systems for liquid food products. Current Opinion in Colloid \& Interface Science. 2010; 15(1-2): 61-72.

[33] Mishra N, Yadav KS, Rai VK, Yadav NP. Polysaccharide encrusted multilayered nano-colloidal system of andrographolide for improved hepatoprotection. Aaps Pharmscitech. 2017; 18(2): 381-92.

[34] Benjamin O, Silcock P, Leus M, Everett DW. Multilayer emulsions as delivery systems for controlled release of volatile compounds using pH and salt triggers. Food Hydrocolloids. 2012; 27(1): 109-18.

[35] Langella A, Calcagno V, De Gregorio V, Urciuolo F, Imparato G, Vecchione R, Netti PA. In vitro study of intestinal epithelial interaction with engineered oil in water nanoemulsions conveying curcumin. Colloids and Surfaces B: Biointerfaces. 2018; 164: 232-9.

[36] Guzey D, McClements DJ. Formation, stability and properties of multilayer emulsions for application in the food industry. Advances in colloid and interface science. 2006; 128: 227-48. 